\title{
Supporting Information: Observation of
}

\section{Anomalous Hall Effect in $\mathrm{NdTiO}_{3} / \mathrm{SrTiO}_{3}$}

\section{Heterostructure}

\author{
Prithwijit Mandal, Shashank Kumar Ojha, Ranjan Kumar Patel, Siddharth \\ Kumar, and Srimanta Middey*
}

Department of Physics, Indian Institute of Science, Bengaluru 560012, India

E-mail: smiddey@iisc.ac.in

\section{Growth and characterization}

Fig. S1(a) shows the image of the reflection high electron diffraction (RHEED) pattern of the 4 uc NTO on STO sample. The labels, $(0,0)$ and $(0,-1),(0,1)$ denote the specular and off-specular RHEED spots. These sharp spots confirm excellent crystallinity of the surface of the film. Fig. S1(b) shows the experimentally observed X-ray reflectivity (XRR) data and the fitting, which is done using GENX software for the 20 uc NTO on STO sample. We have found that the surface roughness is $2.3 \AA$, indicating smooth surface morphology of the film. X-ray diffraction (XRD) data (Fig. S1(c)) shows that the film peak and the substrate peak almost coincide with each other; it arises due to very small lattice mismatch between the substrate and film. 

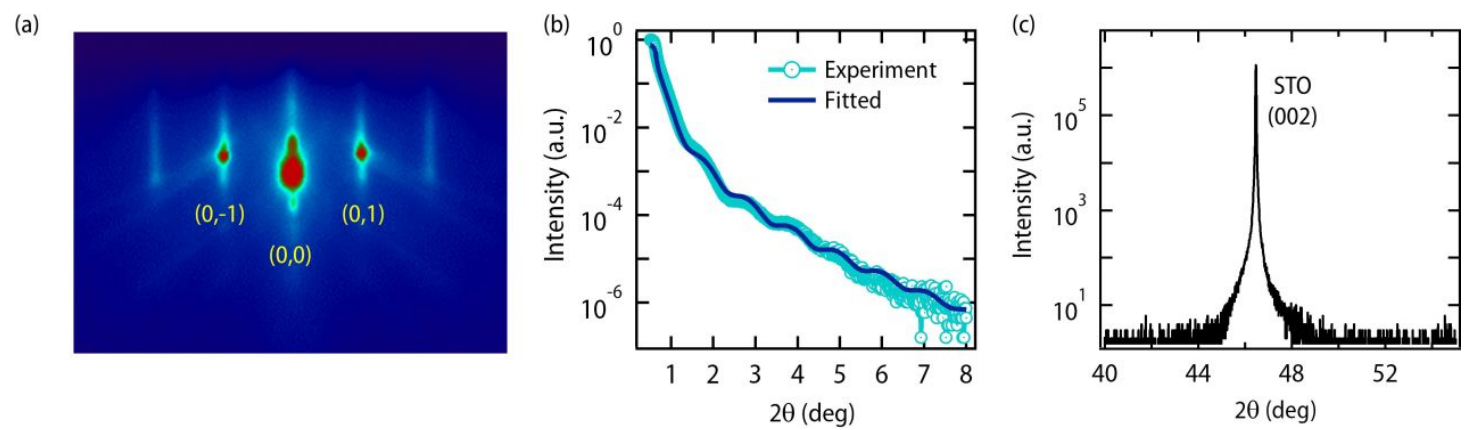

Figure S1: (a) RHEED image of 4 uc NTO on STO sample. (b) and (c) are XRR along with the corresponding fitting and XRD data respectively for 20 uc NTO on STO sample.

\section{Annealing experiment on the film}

The transport data (Fig. S2) for the as grown 10 uc NTO on STO film shows highly metallic behavior. In contrast, highly insulating behavior is observed after annealing it in $\mathrm{O}_{2}$ atmosphere at $400^{\circ} \mathrm{C}$ for 6 hours. Such metal to insulator transition indicates that the oxygen vacancies play a crucial role in the metallic behavior of the samples.

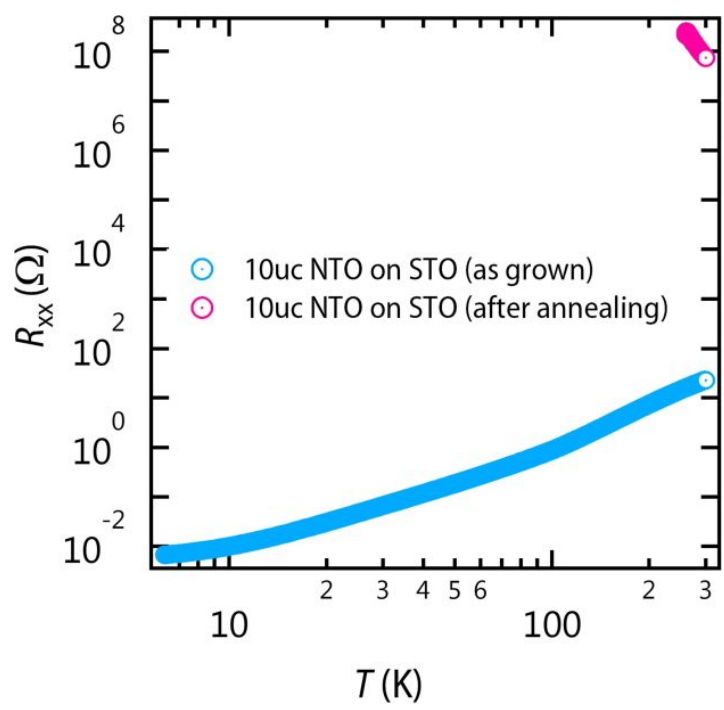

Figure S2: Longitudinal resistance $\left(R_{x x}\right)$ as a function of temperature $(T)$ for as grown and $\mathrm{O}_{2}$ annealed 10 uc NTO on STO samples. 


\section{Growth of amorphous NTO on STO}

Fig. S3 shows the sheet resistance vs. temperature of a $4 \mathrm{~nm}$ NTO amorphous film on STO substrate. The growth was carried out at room temperature. The amorphous nature of the film is confirmed by the absence of any diffraction pattern in the RHEED image. The sheet resistance $\left(R_{S}\right)$ vs temperature $(T)$ plot shows metallic behavior of the sample. $R_{S}$ increases by a factor of $10^{2}$ compared to a $10 \mathrm{uc}$ NTO sample grown at high temperature. The carrier density at $300 \mathrm{~K}$ also reduces by almost a factor of $10^{2}$. Below $80 \mathrm{~K}$, we have observed nonlinearity in the Hall data due to multi-carrier transport. Using the two carrier model, we have extracted the Hall mobility $(\mu)$ and sheet carrier density $(n)$ (inset). It clearly shows the presence of two type of carriers: high mobility with low sheet carrier density and low mobility with high sheet carrier density.

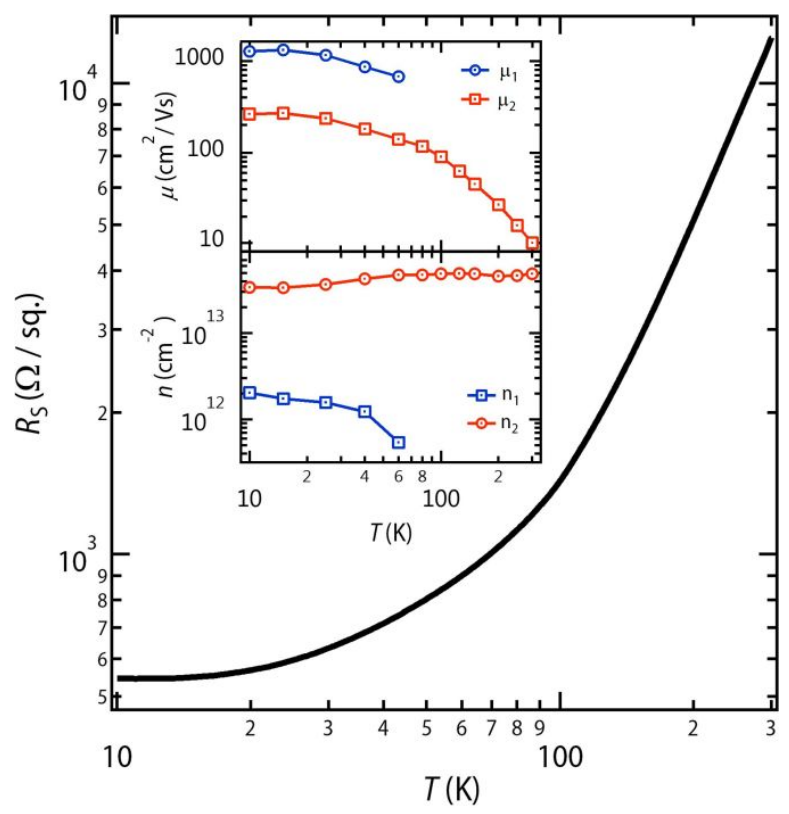

Figure S3: $R_{S}$ vs $T$ plot for the amorphous NTO on STO sample. The inset shows the mobility and sheet carrier density extracted from the Hall measurement. The error in the values of the mobility and sheet carrier density are smaller than the size of the symbols. 


\section{Nonlinearity in the Hall data}

The Hall data at different temperatures for 4 uc and 10 uc NTO on STO samples are shown in Fig. S4(a) and (b) respectively. The Hall coefficient $\left(R_{H}\right)$ for 4 uc NTO on STO (Fig. $\mathrm{S} 4(\mathrm{c})$ ) shows non-linear behavior at low temperature. $R_{H}$ for $10 \mathrm{uc} \mathrm{NTO} / \mathrm{STO}$ has been shown in the main text.
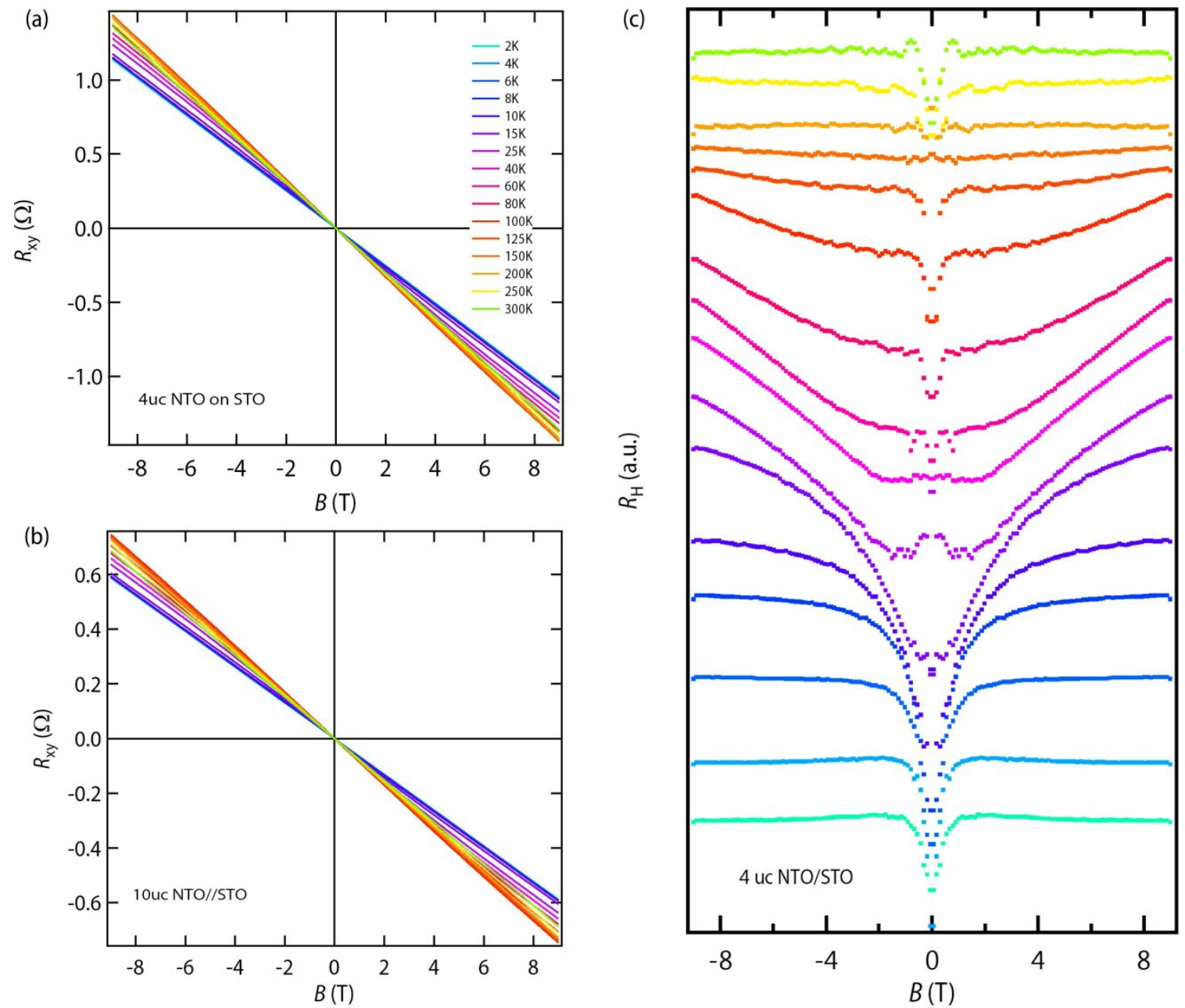

Figure S4: (a), (b) $R_{x y}$ (curves measured at various temperatures) for 4 uc and 10 uc NTO on STO respectively. (c) Hall coefficient $\left(R_{H}=R_{x y} / B\right)$ plotted for the same temperatures. 


\section{Temperature dependence of the mobility}

At higher temperature $(150 \mathrm{~K}$ to $300 \mathrm{~K}), \mu$ varies as $T^{-3}$ (Fig. S5)

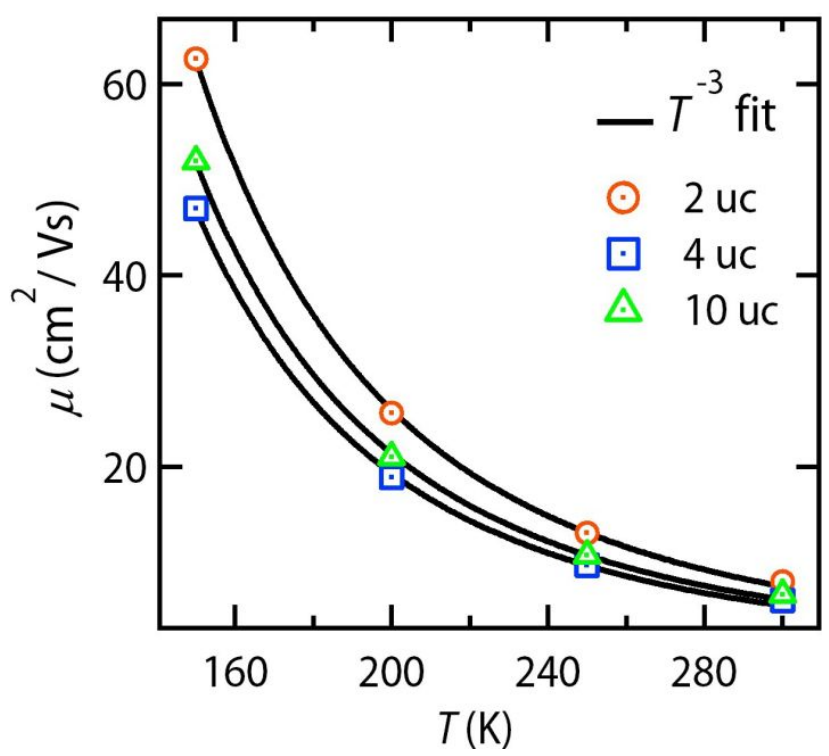

Figure S5: $\mu$ as a function of $T$ and the fitting with $T^{-3}$ function for $2,4,10$ uc NTO on STO samples. 